\title{
Research on New Technology of Semi-steel Dephosphorization in Ladle Based on Water Simulation Experiment
}

\author{
Yonghui Han ${ }^{a}$,Daqiang Cang ${ }^{b}$ \\ School of Metallurgical and Ecological Engineering,University of Science and Technology \\ Beijing,Beijing 100083,China \\ ahebeihyh@126.com, ${ }^{\text {}}$ cangdaqiang@metall.ustb.edu.cn
}

Keywords: ladle;dephosphorization; semi-steel;mixing time;water simulation.

\begin{abstract}
Dephosphorization in the ladle is a new type of dephosphorization method. The water simulation experiment of semi-steel dephosphorization in 100t ladle of Chengde steel has been carried out.The best mixing time has been investigated for various process parameters of blowing oxygen lance and bottom blowing.It turns out to be that the best location of the pure bottom blowing is $0.7 \mathrm{R}-120^{\circ}$,the best bottom blowing flow rate is $100 \mathrm{~L} / \mathrm{min}$; and the best of the top bottom blowing rate is $3000 \mathrm{Nm}^{3} / \mathrm{h}$,top gun angle is $60^{\circ}$, top lance height is $500 \mathrm{~mm}$. The order of three elements on influencing the mixing time is as follows:Top blowing flow $>$ top gun height $>$ top gun angle.The average of dephosphorus efficiency is $51.8 \%$ in industrial experiments.
\end{abstract}

\section{Introduction}

Chengde area is rich in vanadium titanium magnetite resources.Semi-steel has higher phosphorus rate,in order to reduce BOF load,semi-steel dephosphorization pretreatment is necessary.Converter tapping process has a relatively short time,generally 4-6 minutes.Using semi-steel dephosphorization in ladle is a new dephosphorization technology.The use of oxygen top blowing gun and bottom argon blowing can create a dynamic condition for dephosphorization reaction,which made good effects of dephosphorization ${ }^{[1]}$.Among them,the mixing time is one of the main reference indexes ${ }^{[2]}$. In this paper,by analyzing the water model,we can find the shortest time to satisfy dephosphorization reaction speed and argon blowing in ladle cycle.

\section{Establishment of Model}

According to the similarity principle,physical model established in this experiment of $100 \mathrm{t}$ ladle.The geometric model and the prototype remain similar and make sure that the geometric similarity ratio is $4: 1$.The geometric size of prototype and model is shown in table1.

Table 1. Geometric parameters of prototype and model

\begin{tabular}{ccc}
\hline & Prototype/mm & Model $/ \mathrm{mm}$ \\
\hline Top diameter & 2900 & 725 \\
Bottom diameter & 2482 & 620.5 \\
Deep of furnace & 3520 & 880 \\
Height of liquid level & 2860 & 715 \\
Thickness of slag layer & 60 & 15 \\
\hline
\end{tabular}

Determination of the gas parameters.In the calculation of gas flow in ladle in this experiment,the bottom blowing gas of prototype is argon,the model gas is nitrogen;the top blowing gas of prototype and model are both oxygen.The calculated numerical flow of bottom blowing and top blowing gas of the model are shown in table2-3.

Selection of the model of slag medium.According to the similarity principle,water model experiment needs to meet the equal kinetic parameter We.For the simulation of slag layer,simulation mediumshould be selected according to the calculation results of the number of We.Select liquid paraffin as the steel slag simulated medium. 
Table 2. Bottom blowing gas flow of prototype and model

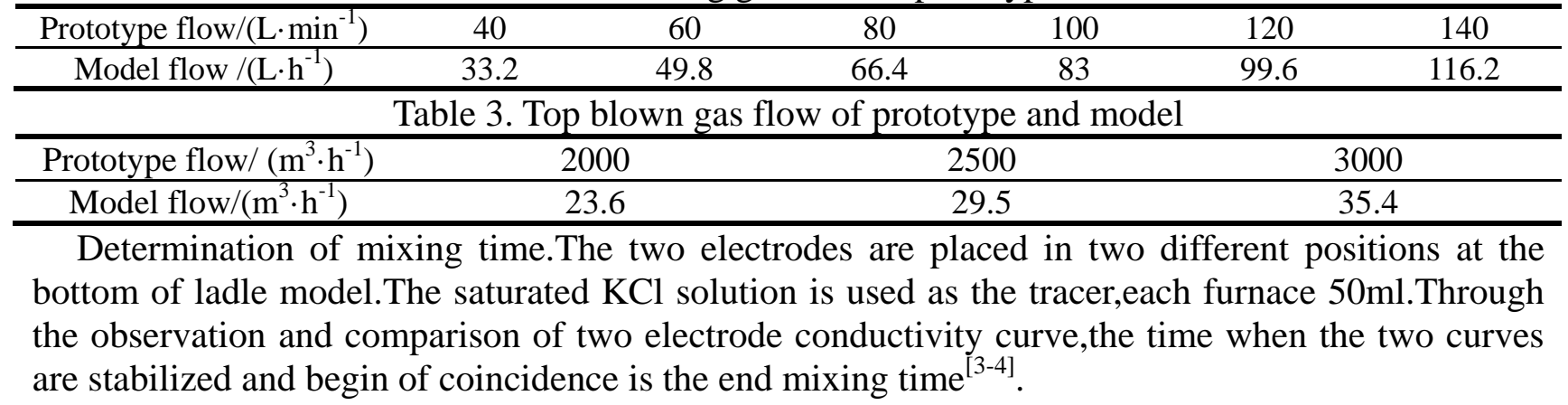

\section{Experiment result and analysis}

\subsection{Determination of bottom blowing position}

The bottom blowing is a constant of $80 \mathrm{~L} / \mathrm{min}$,and mixing times are shown in table4.

Table 4. Mixing time of different bottom blowing

\begin{tabular}{|c|c|c|c|c|c|c|c|c|c|c|c|c|c|c|c|c|}
\hline Radii & \multicolumn{4}{|c|}{$0.4 \mathrm{R}$} & \multicolumn{4}{|c|}{$0.5 \mathrm{R}$} & \multicolumn{4}{|c|}{$0.6 \mathrm{R}$} & \multicolumn{4}{|c|}{$0.7 \mathrm{R}$} \\
\hline Angle $/^{\circ}$ & 60 & 90 & 120 & 180 & 60 & 90 & 120 & 180 & 60 & 90 & 120 & 180 & 60 & 90 & 120 & 180 \\
\hline $\begin{array}{c}\text { Mixing } \\
\text { time/s }\end{array}$ & 81 & 69 & 60 & 92 & 69 & 80 & 74 & 86 & 81 & 70 & 59 & 72 & 66 & 62 & 60 & 75 \\
\hline
\end{tabular}

From Table4, we can know that when the two flow streams are close to each other,the mixing time is generally longer.The main reason is that the interaction process of two streams in the rise of stirring liquid steel,which cancels each other out of a part of the mixing energy.when the two streams flow distance is too far, the mixing time is also relatively longer.More obvious scheme is that when the angle of two holes'radii is $180^{\circ}$,mixing time is generally longer than other schemes. The mixing time of $0.7 \mathrm{R}-120^{\circ}$ is the shortest,which means the scheme is the best bottom blowing scheme.From figure1 and figure2,We can ultimately ensure that the best bottom blowing scheme is $0.7 \mathrm{R}-120^{\circ}$.

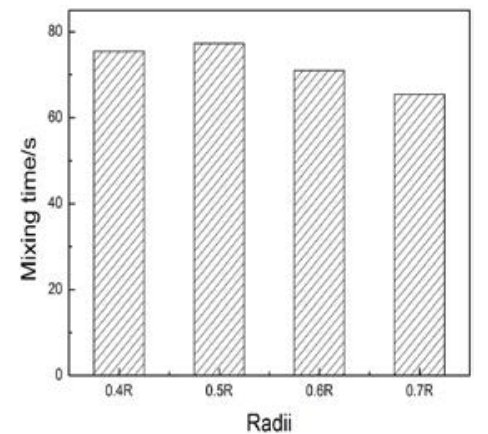

Figure 1. Average mixing time at different radii

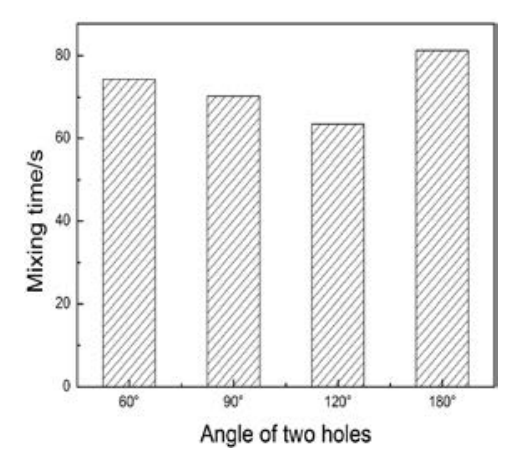

Figure 2. Average mixing time at different angle

\subsection{Determination of bottom blowing flow}

Through the changes at different bottom blowing flow rate to the effects of mixing time based on the actual situation to determine the best bottom blowing flow rate.

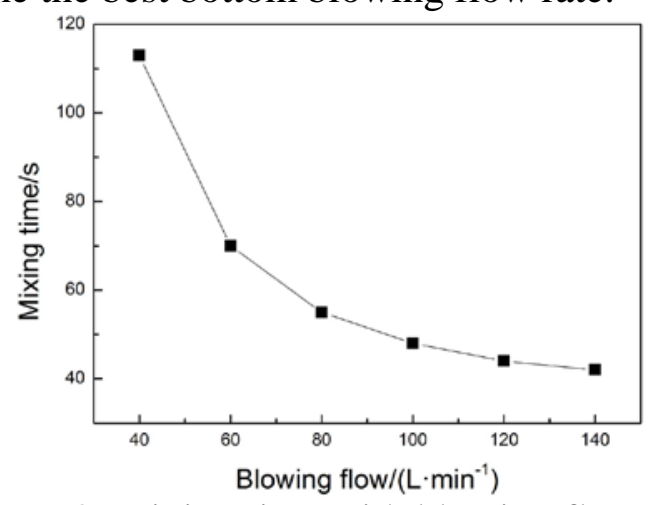

Figure 3. Mixing time with blowing flow rate 
Analysis to Figure 3 shows that the mixing time reduces with increasing bottom blowing flow rate.Combining the actual comprehensive on-site factors we ultimately selected bottom blowing flow rate as $100 \mathrm{~L} / \mathrm{min}$.

\subsection{Analysis of orthogonal experiments}

After reaching the optimal bottom blowing location $0.7 \mathrm{R}-120^{\circ}$ and bottom blowing flow rate $100 \mathrm{~L} / \mathrm{min}$,the orthogonal test has been taken for the research on the mixing time.Combining with the two top lances, we did the orthogonal experiment,and obtained the flow of top blowing flow,top gun angle, impact of top lance height on the mixing time,see table5.

Table 5. Factor level

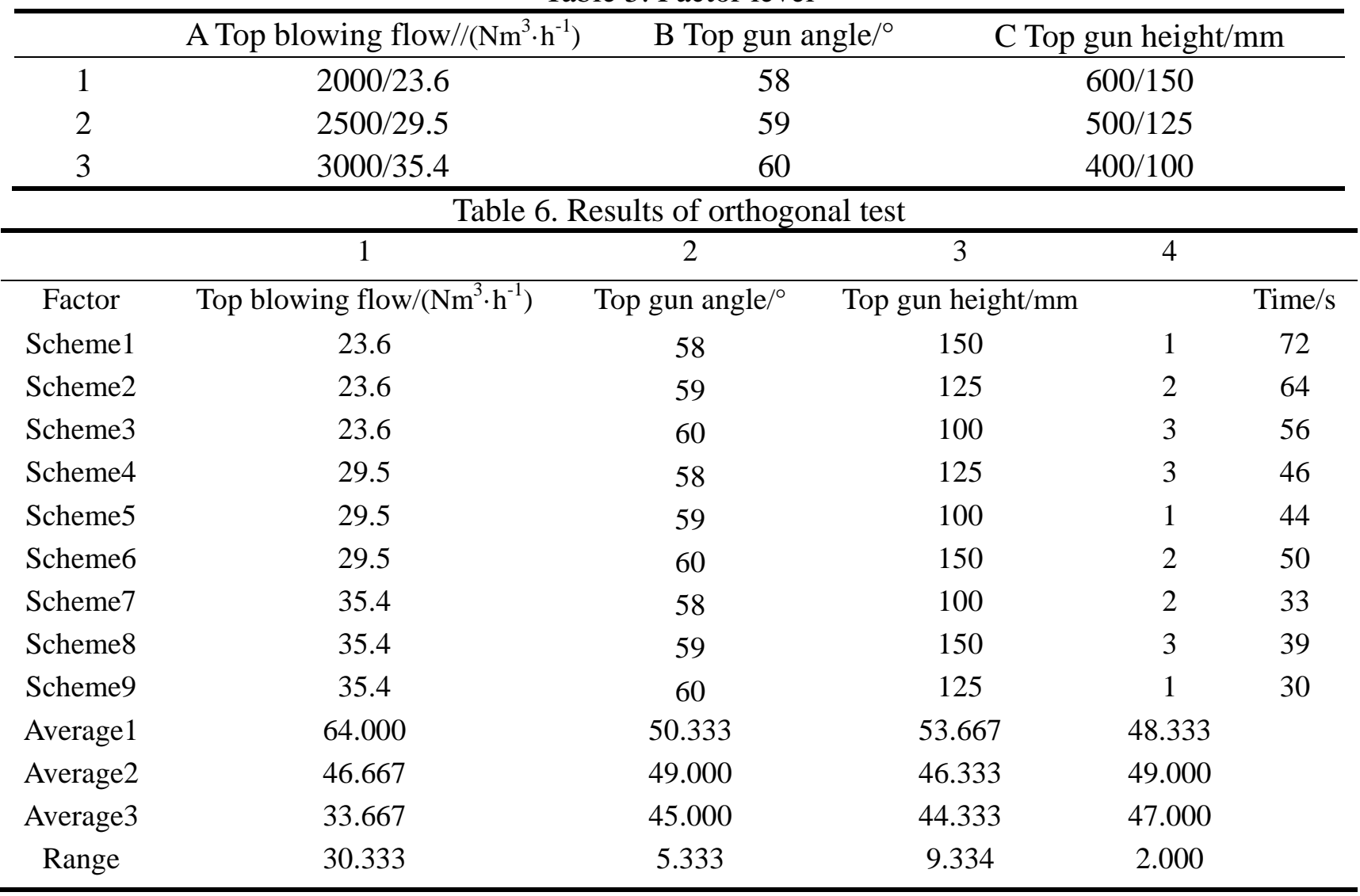

Table6 is orthogonal experiment results in the bottom position $0.7 \mathrm{R}-120^{\circ}$,bottom blowing flow rate of $100 \mathrm{~L} / \mathrm{min}$. The order of factors is top flow,top gun angle,and top lance height.

From table6,the smallest top blowing flow rate in the orthogonal experiment of mixing time is $35.4 \mathrm{Nm}^{3} / \mathrm{h}$,top lance height is $125 \mathrm{~mm}$, top gun angle is $60^{\circ}$,corresponding to the actual injection scheme that top blowing flow rate is $3000 \mathrm{Nm}^{3} / \mathrm{h}$,top lance height is $500 \mathrm{~mm}$,and top gun angle is $60^{\circ}$,and the lowest mixing time is 30s. The order of three factors of effect on liquid steel stirring was blown flow $>$ top lance height $>$ top gun angle.

\section{Industrial Experiments}

Industrial Experiments were done by 20 furnaces in 100t ladle.Dephosphorization slag was added in two batches.Oxygen blowing started when steel pouring 5 7s. Scheme of semi steel dephosphorization was shown in table7.

Table 7. Scheme of semi steel dephosphorization

\begin{tabular}{cccccc}
\hline $\mathrm{CaO} / \mathrm{kg}$ & $\mathrm{CaF}_{2} / \mathrm{kg}$ & $\mathrm{FetO} / \mathrm{kg}$ & Slag $/ \mathrm{kg}$ & Oxygen flow $/\left(\mathrm{Nm}^{3} \cdot \mathrm{h}^{-1}\right)$ & Oxygen Time $/ \mathrm{min}$ \\
\hline 500 & 210 & 690 & 1400 & 3000 & 4 \\
\hline
\end{tabular}

Argon was blown by ladle bottom blowing two points (radius $0.7 \mathrm{R}$, angle $120^{\circ}$ ), bottom blowing amount is $100 \mathrm{~L} \cdot \mathrm{min}^{-1}$. Dephosphorization lance design parameters was shown in table8. 
Table 8. Main design parameters of dephosphorization lance

\begin{tabular}{ccccccc}
\hline $\begin{array}{c}\text { Hole } \\
\text { numbe } \\
\mathrm{r}\end{array}$ & Mach & $\begin{array}{c}\text { Oxygen flow } \\
/\left(\mathrm{Nm}^{3} \cdot \mathrm{h}^{-1}\right)\end{array}$ & Height/mm & Angle ${ }^{\circ}$ & $\begin{array}{c}\text { Throat diameter } \\
/ \mathrm{mm}\end{array}$ & $\begin{array}{c}\text { Outlet } \\
\text { diameter } \\
/ \mathrm{mm}\end{array}$ \\
\hline 1 & 1.4 & 3000 & 500 & 60 & 45 & 48 \\
\hline
\end{tabular}

Industrial experiments show that dephosphorization slag had a good performance in slag melting and degree of foaming is higher.The arrangement of dephosphorization oxygen blowing lance and bottom blowing is more reasonable.Oxygen utilization rate is better,the slag had moderate content of $\mathrm{FeO}$.The average dephosphorization rate is $51.8 \%$.Dephosphorization results were shown in figure4.

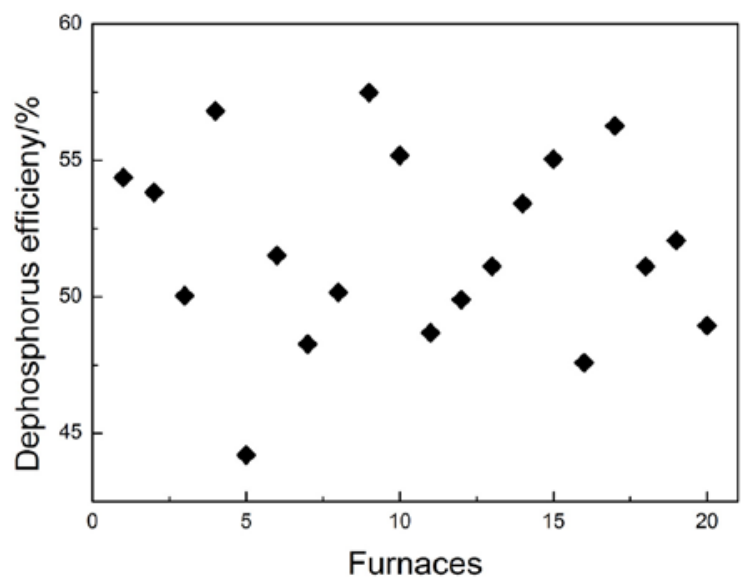

Figure 4. Dephosphorization results

\section{Conclusions}

(1)Through the pure bottom blowing experiments to yield the best bottom blowing location and bottom blowing flow rate that match the field.Bottom blowing location is $0.7 \mathrm{R}-120^{\circ}$, while the best bottom blowing flow rate is $100 \mathrm{~L} / \mathrm{min}$.

(2)Through the orthogonal experiment of top and bottom blowing to get optimal blowing program.Top blowing flow is $3000 \mathrm{Nm}^{3} / \mathrm{h}$, top gun angle is $60^{\circ}$, and top lance height $500 \mathrm{~mm}$.

(3)The order of elements influencing the mixing time is:topblowing flow $>$ top lance height $>$ top gun angle.

(4)Industrial experiments show that the arrangement of dephosphorization oxygen blowing lance and bottom blowing is more reasonable.The average of dephosphorus efficiency is $51.8 \%$.

\section{Acknowledgements}

The corresponding author of this paper is Han Yonghui.This paper is supported by Hebei provincial science and technology support program(12215634D).

\section{References}

[1] Ruiguo Bai.Vanadium resource extraction research and development and utilization technology of Chengde steel [J].Metallurgy of Hebei Province,2012,(02):7-11.

[2] Jing Li,Yanguo Lu,Zhen Cao,et al.Ladle bottom blowing on the water model experiment study on mixing effect.The Seventeenth National Conference on steelmaking works,2013:455-459.

[3] Han J W,Heo S H.Transient Fluid Flow Phenomena in a Gas Stirrred Liquid Bath with Top Oil Layer-Approach Numeriacal Simulation and Water Model Experiments[J].ISSJ International.2001.41(10):1165-1172.

[4] Jonsson L.Modeling of Fluid Flow Conditons Around the Slag/Metal Interface in a Gas-stirred Ladle[J].ISIJ International.1996.36(9):1127-1134. 